On certain theorems in Continued Fractions equivalent to Riemann's and other Transformations of the P-function.

\author{
By E. Lindsay Ince.
}

(Read 8th May 1914. Received 21st May 1914).

\title{
\$. Introduction.
}

The transformation of Continued Fractions into one another is a subject in which very little work has so far been done. Beyond the simple transformations given in works on elementary algebra, few transformation-theorems are known; the best known being those connected with the "contraction" and "extension" of Continued Fractions, and the transformations of Euler, Bauer and Muir.

The purpose of the following paper is to add to the number of known transformations. The method used depends on the fact that every linear differential equation of the second order leads to a continued fraction, so that theorems involving the transformation of one such differential equation into another would be expected to lead to transformations of continued fractions hitherto unknown.

\section{\$2. The General Method.}

$$
\text { Let } \frac{d^{2} y}{d z^{2}}+p(z) \frac{d y}{d z}+q(z) y=0
$$

be a linear differential equation of the second order; then if we differentiate it with respect to the independent variable $z$ we obtain a linear differential equation of the third order. We may eliminate $y$ between equation (1) and this new equation, and thus obtain an equation of the form

$$
\frac{d^{3} y}{d z^{3}}+p_{1}(z) \frac{d^{2} y}{d z^{2}}+q_{1}(z) \frac{d y}{d z}=0
$$

From this equation we obtain by differentiating with respect to $z$ and eliminating $\frac{d y}{d z}$ a third equation

$$
\frac{d^{4} y}{d z^{4}}+p_{2}(z) \frac{d^{3} y}{d z^{3}}+q_{2}(z) \frac{d^{2} y}{d z^{2}}=0 .
$$


We may continue this process indefinitely; after $n$ successive differentiations and the corresponding eliminations we obtain the equation

$$
\frac{d^{n+2} y}{d z^{n+2}}+p_{n}(z) \frac{d^{n+1} y}{d z^{n+1}}+q_{n}(z) \frac{d^{n} y}{d z^{n}}=0
$$

Now, denoting $\frac{d y}{d z}$ by $y^{\prime} ; \frac{d^{\prime 2} y}{d z^{2}}$ by $y^{\prime \prime}$, and so on, we obtain from equation (1) the relation

$$
\frac{y^{\prime}}{y}=-\frac{q}{p+\frac{y^{\prime \prime}}{y^{\prime}}} .
$$

But $\frac{y^{\prime \prime}}{y^{\prime}}$ may be obtained from equation (2) in a similar form, viz.

$$
\frac{y^{\prime \prime}}{y^{\prime}}=\frac{-q_{1}}{p_{1}+\frac{y^{\prime \prime \prime}}{y^{\prime \prime}}},
$$

and thus we get the relation

$$
\frac{y^{\prime}}{y}=\frac{-q}{p-\frac{q_{1}}{p_{1}+\frac{y^{\prime \prime \prime}}{y^{\prime \prime}}}} .
$$

Continuing this process, and making use of the ratios of successive differential coefficients of $y$ deduced from the equation already obtained, we finally get the ratio $\frac{y^{\prime}}{y}$ expressed in terms of a continued fraction, viz.

$$
\frac{y^{\prime}}{y}=-\frac{q}{p}-\frac{q_{1}}{p_{2}}-\frac{q_{2}}{p_{2}}-
$$

Next suppose that we apply the transformation

$$
\left.\begin{array}{l}
y=v \phi(x) \\
z=\psi(x)
\end{array}\right\}
$$

to the differential equation (1), and that it is changed thereby into the new equation

$$
\frac{d^{2} v}{d x^{2}}+\mathrm{P}(x) \frac{d v}{d x}+\mathrm{Q}(x) v=0
$$


From this new equation (5), by applying the process above described, we obtain $\frac{v^{\prime}}{v}$ in terms of a continued fraction, thus :

$$
\frac{v^{\prime}}{v}=-\frac{\mathrm{Q}}{\mathrm{P}}-\frac{\mathrm{Q}_{1}}{\mathrm{P}_{1}}-\frac{\mathrm{Q}_{2}}{\mathrm{P}_{2}}-
$$

when the Q's correspond to the $q$ 's of the original. equation and those derived from it by the process of differentiaticon and elimination, and the P's in like manner to the $p$ 's.

Thus the transformation (4) converts the continued fraction of equation (3) into that of equation (6), always provided, however, that $v$ is the same solution of equation (5) as $y$ is of equation (1). Thus if we denote the first continued fraction by $F$, we have, applying the transformation (4),

$$
\begin{gathered}
\mathrm{F}=\frac{1}{y} \frac{d y}{d z}=\frac{1}{v \phi}\left(\frac{v^{\prime} \phi+v \phi^{\prime}}{\psi^{\prime}}\right) \\
=\frac{1}{\psi^{\prime}}\left(\frac{v^{\prime}}{v}+\frac{\phi^{\prime}}{\phi}\right),
\end{gathered}
$$

dashes denoting differentiation with respect to the new independent variable $x$.

Now $\frac{v^{\prime}}{v}$ is the continued fraction obtained frona the transformed differential equation (5) and may be denoted by $\mathrm{G}$.

Then we have the relation

$$
\begin{aligned}
& \mathbf{F}=\frac{1}{\psi^{\prime}}\left(\mathrm{G}+\frac{\phi^{\prime}}{\phi}\right) \\
& \mathrm{G}=\mathbf{F} \psi^{\prime}-\frac{\phi^{\prime}}{\phi} . \cdots
\end{aligned}
$$

or

The question as to whether $v$ and $y$ are or are not the same solution of the transformed and the original differential equation will be discussed subsequently in the different cases.

\section{\$3. Application to equations of hypergeometric type.}

In general, however, the successive differential equations become more and more involved, so that it becomes extremely difficult to obtain a general expression for the $n^{\text {th }}$ derived equation.

Nevertheless-in certain simple cases we may proceed without difficulty to the $n^{\text {th }}$ derived equation and obtain the corresponding continued fraction. 
Such a case arises in connection with the differential equation

$$
\left(a z^{2}+b z+c\right) \frac{d^{2} y}{d z^{2}}+(e z+f) \frac{d y}{d z}+g y=0,
$$

where $a, b, c, e, f$ and $g$ are constants.

If we differentiate this with respect to the independent variable $z$ we obtain the equation

$$
\left(a z^{2}+b z+c\right) \frac{d^{3} y}{d z^{3}}+\{(2 a+e) z+(b+f)\} \frac{d^{2} y}{d z^{2}}+(e+g) \frac{d y}{d z}=0
$$

in which the dependent variable $y$ does not appear and no elimination is necessary.

By differentiating equation (8) $n$ times we finally arrive at the equation

$$
\begin{aligned}
\left(a z^{2}+b z+c\right) \frac{d^{n+2} y}{d z^{n+2}} & +\{(2 n a+e) z+(n b+f)\} \frac{d^{n+1} y}{d z^{n+1}} \\
+ & \{n(n-1) a+n e+g\} \frac{d^{n} y}{d z^{n}}=0 .
\end{aligned}
$$

These equations lead directly to the relation

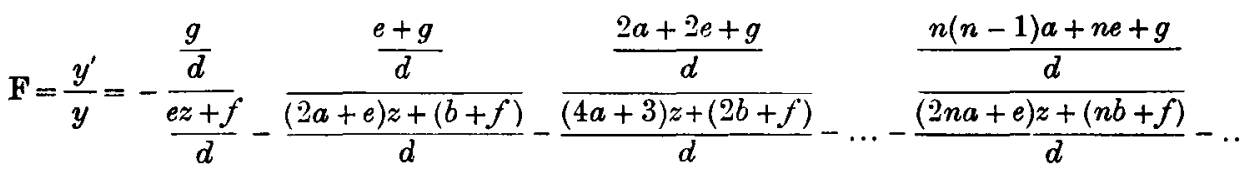

$$
\begin{aligned}
& =\frac{-g}{e z+f}-\frac{(e+g) d}{(2 a+e) z+(b+f)}-\frac{(2 a+2 e+g) d}{(4 a+e) z+(2 b+f)}-\ldots-\frac{\{n(n-1) a+n e+g\} d}{(2 n a+e) z+(n b+f)} \\
& \text { where } d \equiv a z^{2}+b z+c \text {. }
\end{aligned}
$$

In order that this continued fraction may converge and thus be of any practical utility, it is necessary that in the equation

$$
\left(a z^{2}+b z+c\right) \frac{d^{2} y}{d z^{2}}+(e z+f) \frac{d y}{d z}+g y=0
$$

the first term $a z^{2}+b z+c \equiv d$ should be of less importance than the other two, so that a good approximation is obtained by discarding it, in which case a first approximation to its value is

$$
\frac{1}{y} \frac{d y}{d z}=-\frac{g}{e z+f}
$$

Thus in the case of the ordinary hypergeometric equation

$$
z(z-1) \frac{d^{2} y}{d z^{2}}+\{-c+(a+b+1) z\} \frac{d y}{d z}+a b y=0,
$$


where $z$ is small (i.e. near the singularity 0 ), the two last terms are non-zero, but the first term tends to zero when $z$ tends to zero, so that here the condition is satisfied.

\section{§4. Nature of the solution $y$ in the preceding section.}

In order to determine which solution of the differential equation (8) $y$ happens to be, we may regard the matter from the point of view of the Riemann P-function, which is defined as being any solution of the generalised hypergeometric equation

$$
\begin{array}{r}
\frac{d^{\prime} y}{d z^{2}}+\left\{\frac{1-\alpha-\alpha^{\prime}}{z-a}+\frac{1-\beta-\beta^{\prime}}{z-b}+\frac{1-\gamma-\gamma^{\prime}}{z-c}\right\} \frac{d y}{d z} \\
+\left\{\frac{\alpha \alpha^{\prime}(a-b)(a-c)}{z-a}+\frac{\beta \beta^{\prime}(b-c)(b-a)}{z-b}+\frac{\gamma \gamma^{\prime}(c-a)(c-b)}{z-c}\right\} \\
\frac{y}{(z-a)(z-b)(z-c)}=0
\end{array}
$$

and is denoted by

$$
\mathbf{P}\left\{\begin{array}{llll}
a & b & c & \\
\alpha & \beta & \gamma & z \\
\alpha^{\prime} & \beta^{\prime} & \gamma^{\prime} &
\end{array}\right\} .
$$

The generalised hypergeometric equation has three regular singular points $a, b, c$, with exponents $\alpha$ and $\alpha^{\prime}$ at $a, \beta$ and $\beta^{\prime}$ at $b$, and $\gamma$ and $\gamma^{\prime}$ at $c$, respectively.

The P-function corresponding to the differential equation (8) is of the form

$$
\mathbf{P}\left\{\begin{array}{cccc}
\lambda & \infty & \mu & \\
\alpha & \beta & \gamma & z \\
\alpha^{\prime} & \beta^{\prime} & \gamma^{\prime} &
\end{array}\right\}
$$

where $\lambda$ and $\mu$ are the roots of the equation

$$
a z^{2}+b z+c=0 \text {. }
$$

Near the value $\lambda$ of $z$, there will be two distinct expansions of $y$.

One, viz. $y_{1}$, is

$$
y_{1}=(z-\lambda)^{a}+\mathbf{A}(z-\lambda)^{a+1}+\ldots
$$

whence

$$
y_{1}^{\prime}=\alpha(z-\lambda)^{a-1}+(\alpha+1) \mathbf{A}(z-\lambda)^{a}+\ldots
$$

and

$$
\frac{y_{1}^{\prime}}{y_{1}}=\frac{\alpha}{z-\lambda}+\mathrm{K}+\mathrm{L}(z-\lambda)+\mathrm{M}(z-\lambda)^{2}+\mathrm{N}(z-\lambda)^{3}+\ldots
$$


For the other, $y_{1}$, we shall have

$$
\frac{y_{2}^{\prime}}{y_{2}}=\frac{\alpha^{\prime}}{z-\lambda}+\mathbf{K}^{\prime}+\mathbf{L}^{\prime}(z-\lambda)+\ldots
$$

The other notable solutions are as follows :

At infinity,

$$
y_{3}=\frac{1}{z^{\beta}}+\frac{A}{z^{\beta+1}}+\frac{B}{z^{\beta+2}}+\ldots .
$$

For this

$$
y_{3}^{\prime}=-\frac{\beta}{z^{\beta+1}}+\ldots
$$

and

$$
\frac{y_{3}^{\prime}}{y_{3}}=-\frac{\beta}{z}+\frac{\mathbf{K}^{\prime \prime}}{z^{2}}+\frac{\mathbf{L}^{\prime \prime}}{z^{3}}+\frac{\mathbf{M}^{\prime \prime}}{z^{4}}+\ldots,
$$

so also $\quad \frac{y_{4}^{\prime}}{y_{4}}=-\frac{\beta^{\prime}}{z}+\frac{\mathbf{K}^{\prime \prime \prime}}{z^{2}}+\frac{L^{\prime \prime \prime}}{z^{3}}+\frac{\mathbf{M}^{\prime \prime \prime}}{z^{4}}+\ldots$.

Near $z=\mu$, we have, in like manner,

$$
\frac{y_{s}^{\prime}}{y_{s}}=\frac{\gamma}{z-c}+\ldots
$$

and

$$
\frac{y_{6}^{\prime}}{y_{6}}=\frac{\gamma^{\prime}}{z-c}+\ldots
$$

Our purpose is now to find out which of these ratios, viz.,

$$
\frac{y_{1}^{\prime}}{y_{1}}, \frac{y_{2}^{\prime}}{y_{2}} \ldots \frac{y_{6}^{\prime}}{y_{6}},
$$

is represented by the continued fraction we have obtained.

Now any equation of the form (8) may be reduced to the form of the ordinary hypergeometric equation,

$$
z(z-1) \frac{d^{2} y}{d z^{2}}+\{-\gamma+(\alpha+\beta+1) z\} \frac{d y}{d z}+\alpha \beta y=0
$$

by moving the singularities to the points $z=0$ and $z=1$, and making zero one of the exponents $\alpha$ and $\alpha^{\prime}$ and one of $\gamma$ and $\gamma^{\prime}$.

The corresponding P-function is

$$
\mathbf{P}\left\{\begin{array}{ccc}
0 & \infty & 1 \\
0 & \alpha & 0 \\
1-\gamma & \beta & \gamma-\alpha-\beta
\end{array}\right\}
$$


and the six notable solutions and the corresponding ratios $\frac{y^{\prime}}{y}$ are

i. $y_{1}=1+\frac{\alpha \beta}{\gamma} z+\ldots \ldots \ldots \ldots \ldots . \quad \frac{y_{1}}{y_{1}}=\frac{\alpha \beta}{\gamma}+$

ii. $y_{2}=z^{1-\gamma}+\mathbf{A} z^{-\gamma}+\ldots \ldots \ldots . \quad \frac{y_{2}}{y_{2}}=\frac{1-\gamma}{z}+$

iii. $y_{3}=\frac{1}{z^{a}}+\frac{A_{1}}{z^{a+1}}+\ldots \ldots . . \quad \frac{y_{3}}{y_{3}}=-\frac{\alpha}{z}+$

iv. $y_{4}=\frac{1}{z \beta}+\frac{A_{2}}{z \beta+1}+\ldots \ldots . . \frac{y_{4}}{y_{4}}=-\frac{\beta}{z}+\ldots$

v. $y_{5}=1+\frac{\alpha \beta}{\gamma-\alpha-\beta-1}(z-1)+. . \frac{y_{5}}{y_{5}}=\frac{\alpha \beta}{\gamma-\alpha-\beta 1}+$

vi. $y_{6}=(z-1)^{\gamma-\alpha-\beta}+\ldots \ldots \ldots \frac{y_{6}}{y_{6}}=\frac{\gamma-\alpha-\beta}{z-1}+\ldots$

Of these six solutions, the solution $y_{1}$ corresponds to the solution $y=\mathrm{F}(\alpha, \beta, \gamma, z)$ of the hypergeometric equation, where $\mathrm{F}(\alpha, \beta, \gamma, z)$ is the hypergeometric series $1+\frac{\alpha \beta}{1 \cdot \gamma} z+\frac{\alpha(\alpha+1) \beta(\beta+1)}{1.2 \cdot \gamma(\gamma+1)} z^{2}+\ldots$

Now a continued fraction corresponding to the hypergeometric equation (11) is

$$
\frac{t^{\prime}}{y}=\frac{-\alpha \beta}{(\alpha+\beta+1) z-\gamma}-\frac{(\alpha+1)(\beta+1) z(z-1)}{(\alpha+\beta+3) z-(\gamma+1)}-\ldots-\frac{(\alpha+n)(\beta+n) z(z-1)}{(\alpha+\beta+2 n+1) z-(\gamma+n)-}
$$

But when $z$ is zero this reduces to $\frac{\alpha \beta}{\gamma}$, and consequently this continued fraction is no other than $\frac{y_{1}}{y_{1}}$ where $y_{1}$ is the solution corresponding to the exponent 0 at the singularity 0 . When $z=1$, it reduces to $\frac{\alpha \beta}{\gamma-\alpha-\beta-1}$, and hence represents $\frac{y_{5}^{\prime}}{y_{5}}$, thus also corresponding to the exponent 0 at the singularity 1 .

\section{\$5. Possible Transformations.}

The problem now arises as to what transformations are applicable, i.e. what transformation would change equation (8) into another equation from which a continued fraction could be completely determined. 
Obviously any transformation

$$
\begin{aligned}
& y=v \phi(x) \\
& z=\psi(x)
\end{aligned}
$$

which changed equation (8) into an equation of the form

$$
\left(\mathrm{A} x^{2}+\mathrm{B} x+\mathrm{C}\right) \frac{d^{2} v}{d x^{2}}+(\mathrm{E} x+\mathrm{F}) \frac{d v}{d x}+\mathrm{G} v=0
$$

would satisfy these conditions, provided always that the solution of this latter equation involved in the continued fraction $\frac{v^{\prime}}{v}$ is that solution which corresponds to the solution of the former equation (8) involved in the continued fraction $\frac{y^{\prime}}{y}$.

To obtain such a transformation, we again have recourse to Riemann's P-function. The P-function corresponding to equation $(8)$ is

where

$$
\begin{gathered}
\mathrm{P}\left\{\begin{array}{ccc}
\lambda & \infty & \mu \\
0 & \beta & 0 \\
\alpha^{\prime} & \beta^{\prime} & \gamma^{\prime}
\end{array}\right\} \\
\alpha^{\prime}=1+\frac{e \lambda+f}{a(\mu-\lambda)} \\
\gamma^{\prime}=1+\frac{e \mu+f}{a(\lambda-\mu)} \\
2 \beta=\frac{e}{a}-1+\frac{\sqrt{e^{2}+a^{2}-2 a e-4 a g}}{a} \\
2 \beta^{\prime}=\frac{e}{a}-1-\frac{\sqrt{e^{2}+a^{2}-2 a e-4 a g}}{a}
\end{gathered}
$$

and $\lambda$ and $\mu$ are as already defined.

Thus any such transformation which changes

into

$$
\mathrm{P}\left\{\begin{array}{llll}
\lambda & \infty & \mu & \\
0 & \beta & 0 & z \\
\alpha^{\prime} & \beta^{\prime} & \gamma^{\prime}
\end{array}\right\}
$$

$$
\mathbf{P}\left\{\begin{array}{llll}
\Lambda & \infty & \mathbf{M} & \\
0 & \mathbf{B} & 0 & x \\
\mathbf{A}^{\prime} & \mathbf{B}^{\prime} & \Gamma^{\prime} &
\end{array}\right\}
$$

is suited to our purpose. Several such transformations are known to exist, and examples of their application are given in the following sections. 


\section{\$6. Riemann's Transformation.}

It is, of course, not necessary to know the transformation itself, provided we are given a relation between two P-functions, for we can construct directly the differential equation corresponding to each of them and at once proceed to the relation between the corresponding continued fractions.

One such relation between two P-functions is given by Riemann, viz.

$$
\mathrm{P}\left\{\begin{array}{lll}
0 & \infty & 1 \\
0 & \beta & 0 \\
\frac{1}{2} & \beta^{\prime} & \gamma^{\prime}
\end{array} z^{2}\right\}=\mathrm{P}\left\{\begin{array}{rlll}
-1 & \infty & 1 & \\
0 & 2 \beta & 0 & z \\
\gamma^{\prime} & 2 \beta^{\prime} & \gamma^{\prime}
\end{array}\right\} \ldots
$$

The P-function on the left hand closely resembles that corresponding to the ordinaly hypergeometric equation

$$
x(x-1) \frac{d^{2} y}{d x^{2}}+\{-c+(a+b+1) x\} \frac{d y}{d x}+a b y=0
$$

provided that for $x$ we write $z^{2}$ and for $c$ we substitute the particular value $\frac{1}{2}$.

Then if $\beta=a, \beta^{\prime}=b$, and $\gamma^{\prime}=\frac{1}{2}-a-b$ the relation becomes

$$
\mathbf{P}\left\{\begin{array}{ccc}
0 & \infty & 1 \\
0 & a & 0 \\
\frac{1}{2} & b & \frac{1}{2}-a-b
\end{array} \quad x\right\}=\mathrm{P}\left\{\begin{array}{ccc}
-1 & \infty & 1 \\
0 & 2 a & 0 \\
\frac{1}{2}-a-b & 2 b & \frac{1}{2}-a-b
\end{array}\right\}
$$

By comparing the right hand member of this equation with the P-function of equation (8) we easily find that it corresponds to the differential equation

$$
\left(z^{2}-1\right) \frac{d^{2} y}{d z^{2}}+(2 a+2 b+1) z \frac{d y}{d z}+4 a b y=0 .
$$

From this we obtain the continued fraction

$$
\frac{1}{y} \frac{d y}{d z}=\frac{-4 a b}{(2 a+2 b+1) z}-\frac{(2 a+1)(2 b+1)\left(z^{2}-1\right)}{(2 a+2 b+3) z}-\ldots-\frac{(2 a+n)(2 b+n)\left(z^{2}-1\right)}{(2 a+2 b+2 n+1) z}
$$

It appears from this expression, and from the differential equation from which it is derived, that this C.F. is not convergent near the origin. In fact, from the corresponding P-function we see that the origin is not a singularity in the case, whereas in the left hand P-function, the origin is a singular point.

Now the continued fraction we derived in a previous section from the hypergeometric equation was shown to correspond to the solution $y=\mathrm{F}(a, b, c, z)$ which is an expansion in the domain of the 
singularity $z=0$, and thus these two continued fractions may not be equivalent in this particular region.

We must therefore find out which of the other five distinct solutions of the hypergeometric equation really is equivalent to the continued fraction (16). This continued fraction is obviously convergent in the domains of the singularities $z=1$ and $z=-1$, so that the appropriate solution of the hypergeometric equation is one which is convergent near one or other of these singular points.

The six distinct solutions of the hypergeometric equation may be denoted by $\mathbf{Y}_{1}, \mathbf{Y}_{\mathrm{n}}, \ldots \mathbf{Y}_{6}$, which is the notation adopted by Forsyth (Differential Equations, $\S 123$ ). Of these $Y_{1}$ and $Y_{2}$ are expansions in the domain of $z-0$ with exponents 0 and $1-c$ respectively; $\mathrm{Y}_{5}$ and $\mathrm{Y}_{6}$ are expansions at infinity with exponents $a$ and $b$; all these are to be excluded. The expansions near the singularity $z=1$ are $Y_{3}$ and $Y_{4}$, the exponents being 0 and $c-a-b$ respectively.

Our continued fraction (16) corresponds to an expansion with exponent 0 at the singularity $z=1$, so that of the solutions of the hypergeometric equation $Y_{3}$ is to be taken.

A typical member of the class $\mathbf{Y}_{3}$ is

which gives

$$
y_{5}=\mathbf{F}(a, b, a+b-c+1,1-x),
$$

$$
\begin{gathered}
-\frac{1}{y_{5}} \frac{d y_{5}}{d x}=\frac{-a b}{(a+b+1)(1-x)-(a+b-c+1)}+\frac{(a+1)(b+1)(}{(a+b+3)(1-x)-(a+1)} \\
\quad \ldots+\frac{(a+n)(b+n)(1-x) x}{(a+b+2 n+1)(1-x)-(a+b-c+n+1)} \\
\quad \ldots-\frac{-a b}{c-(a+b+1) x}-\frac{(a+1)(b+1) x(x-1)}{(c+1)-(a+b+3) x}-\ldots \\
\end{gathered}
$$

where $x=z^{2}$,

or

$$
\begin{gathered}
\frac{-a b}{c-(a+b+1) z^{2}}-\frac{(a+1)(b+1) z^{2}\left(z^{2}-1\right)}{(c+1)-(a+b+3) z^{2}}-\ldots-\frac{(a+n)(b+n) z^{2}\left(z^{2}-1\right)}{(c+n)-(a+b+2 n+1) z^{2}}-\ldots \ldots \ldots \ldots . . . \\
=-\frac{1}{y_{5}} \frac{d y_{5}}{d z} \cdot \frac{1}{2 z}
\end{gathered}
$$


Thus the required relation becomes

$$
\begin{aligned}
& \frac{-a b}{\frac{1}{2}-(a+b+1) z^{2}}-\frac{(a+1)(b+1) z^{2}\left(z^{2}-1\right)}{\frac{3}{2}-(a+b+3) z^{2}}-\ldots-\frac{(a+n)(b+n) z^{2}\left(z^{2}-1\right)}{\frac{\frac{3}{2 n+1}}{2}-(a+b+2 n+1) z^{2}}-\ldots \ldots \ldots \ldots \ldots \ldots \\
& =-\frac{1}{2 z}\left\{\frac{-4 a b}{(2 a+2 b+1) z}-\frac{(2 a+1)(2 b+1)\left(z^{2}-1\right)}{(2 a+2 b+3) z}-\ldots-\frac{(2 a+n)(2 b+n)\left(z^{2}-1\right)}{(2 a+2 b+2 n+1) z}-\ldots\right\}
\end{aligned}
$$

which is convergent in the domain of the singularity $z^{2}=1$.

\section{\$7. Another Transformation of the P-function.}

We have seen ( $\$ 5$ ) that the P-function corresponding to the equation

$$
\left(a z^{2}+b z+c\right) \frac{d^{2} y}{d z}+(e z+f) \frac{d y}{d z}+g y=0
$$

is

$$
\mathbf{P}\left\{\begin{array}{ccc}
\lambda & \infty & \mu \\
0 & \beta & 0 \\
1+\frac{e \lambda+f}{a(\mu-\lambda)} & \beta^{\prime} & 1+\frac{e \mu+f}{a(\lambda-\mu)}
\end{array}\right\} .
$$

The transformation of the dependent variable from $y$ to $u$ by the substitution

$$
y=\left(\frac{z-\lambda}{z-\mu}\right)^{\delta} u
$$

gives us the relation

$$
\begin{aligned}
& \left(\frac{z-\lambda}{z-\mu}\right)^{\delta} \mathrm{P}\left\{\begin{array}{ccc}
\lambda & \infty & \mu \\
0 & \beta & 0 \\
1+\frac{e \lambda+f}{a(\mu-\lambda)} & \beta^{\prime} & 1+\frac{e \mu+f}{a(\lambda-\mu)}
\end{array}\right\} \\
& =\mathrm{P}\left\{\begin{array}{ccc}
\lambda & \infty & \mu \\
\delta & \beta & -\delta
\end{array}\right\}
\end{aligned}
$$

This second P-function is of the same form as the first if

and

$$
\begin{aligned}
& 1+\frac{e \lambda+f}{a(\mu-\lambda)}+\delta=0 \\
& 1+\frac{e \mu+f}{a(\lambda-\mu)}-\delta=0
\end{aligned}
$$


and these conditions are satisfied if

$$
\begin{aligned}
& e=2 a, \\
& f=-a(\lambda+\mu)+a \delta(\lambda-\mu) .
\end{aligned}
$$

When $e$ and $f$ have these particular values we are able to deduce a continued fraction-relation equivalent to the relation between the two P-functions.

The equation (8) becomes, on substituting for $e$ and $f$ their values as given above,

$$
\left(a z^{2}+b z+c\right) \frac{d^{2} y}{d z^{2}}+\{2 a z-a(\lambda+\mu)+a \delta(\lambda-\mu)\} \frac{d y}{d z}+g y=0,
$$

from which we obtain the relation

$$
\begin{aligned}
& \frac{1}{y} \frac{d y}{d z}=\frac{-g}{2 a z+a \delta(\lambda-\mu)-a(\lambda+\mu)}-\frac{(2 a+g) a(z-\lambda)(c-\mu)}{4 a z+a \delta(\lambda-\mu)-2 a(\lambda+\mu)}-\ldots \ldots \\
& -\frac{\{n(n+1) a+g\} a(z-\lambda)(z-\mu)}{2(n+1) a z+a \delta(\lambda-\mu)-(n+1) a(\lambda+\mu)}-\ldots
\end{aligned}
$$

The transformed P-function has the form

$$
\mathbf{P}\left\{\begin{array}{cccc}
\lambda & \infty & \mu & \\
\delta & \beta & -\delta & z \\
0 & \beta^{\prime} & 0 &
\end{array}\right\}
$$

or

$$
\mathrm{P}\left\{\begin{array}{ccc}
\lambda & \infty & \mu \\
0 & \beta^{\prime} & 0 \\
1+\frac{e \mu+f}{a(\lambda-\mu)} & \beta & 1+\frac{e \lambda+f}{a(\mu-\lambda)}
\end{array} \quad z\right\}
$$

the equation corresponding to which is

$$
\left(a z^{2}+b z+c\right) \frac{d^{2} u}{d z^{2}}+\{2 a z-a(\lambda+\mu)-a \delta(\lambda-\mu)\} \frac{d u}{d z}+g u=0 .
$$

whence we derive the relation

$$
\begin{array}{r}
\frac{1}{u} \frac{d u}{d z}=\frac{-g}{2 a z-a \delta(\lambda-\mu)-a(\lambda+\mu)}-\frac{(2 a+g) a(z-\lambda)(z-\mu)}{4 a z-a \delta(\lambda-\mu)-2 a(\lambda+\mu)}-\ldots \ldots \\
\ldots-\frac{\{n(n+1) a+g ; a(z-\lambda)(z-\mu)}{2(n+1) a z-a \delta(\lambda-\mu)-(n+1) a(\lambda+\mu)}-\ldots \ldots
\end{array}
$$

Now $y=\left(\frac{z-\mu}{z-\lambda}\right)^{\delta} u$. 
Hence

$\log y=\delta \log (z-\mu)-\delta \log (z-\lambda)+\log u$,

and

$$
\begin{aligned}
\frac{1}{y} \frac{d y}{d} & =\frac{\delta}{z-\mu}-\frac{\delta}{z-\lambda}+\frac{1}{u} \frac{d u}{d z} \\
& =\frac{\delta(\mu-\lambda)}{(z-\lambda)(z-\mu)}+\frac{1}{u} \frac{d u}{d z} .
\end{aligned}
$$

Thus we obtain the following relation between two continued fractions

$$
\begin{aligned}
& \frac{-g}{2 a z-a \delta(\lambda-\mu)-a(\lambda+\mu)}- \frac{(2 a+g) a(z-\lambda)(z-\mu)}{4 a z-a \delta(\lambda-\mu)-2 a(\lambda+\mu)}-. . \\
&-\frac{\{n(n+1) a+g\} a(z-\lambda)(z-\mu)}{2(n+1) a z-a \delta(\lambda-\mu)-(n+1) a(\lambda+\mu)}-\ldots \\
&=\frac{\delta(\lambda-\mu)}{(z-\lambda)(z-\mu)}+\frac{-g}{2 a z+a \delta(\lambda-\mu)-a(\lambda+\mu)}-\frac{(2 a+g) a(z-\lambda)(z-\mu)}{4 a z+a \delta(\lambda-\mu)-2 a(\lambda+\mu)-} \\
& \ldots-\frac{\{n(n+1) a+g\} a(z-\lambda)(z-\mu)}{2(n+1) a z+a \delta(\lambda-\mu)-(n+1) a(\lambda+\mu)}-\ldots(21)
\end{aligned}
$$

For the particular case of the ordinary hypergeometric equation

$$
z(z-1) \frac{d^{2} y}{d z^{2}}+\{-z+(\alpha+\beta+1) z\} \frac{d y}{d z}+\alpha \beta y=0
$$

we have

$$
\begin{aligned}
& a=1, \lambda=0, \mu=1, \\
& -\gamma=f=-1-\delta, \\
& \alpha+\beta+1=e=2,
\end{aligned}
$$

and

$$
\alpha \beta=g \text {. }
$$

The relation between the continued fractions now becomes

$$
\begin{gathered}
\frac{-g}{2 z+\delta-1}-\frac{(g+2) z(z-1)}{4 z+\delta-2}-\frac{(g+6) z(z-1)}{6 z+\delta-3}-\ldots-\frac{\{n(n+1)+g\} z(z-1)}{2(n+1) z+\delta-(n+1)}-\ldots \\
=\frac{-\delta}{z(z-1)}+\frac{-g}{2 z-\delta-1}-\frac{(g+2) z(z-1)}{4 z-\delta-2}-\frac{(g+6) z(z-1)}{6 z-\delta-3}-\ldots-\frac{\{n(n+1)+g\} z(z-1)}{2(n+1) z-\delta-(n+1)}-\ldots \\
(g \neq 0)
\end{gathered}
$$

The expansions of the two P-functions involved are the same in the two cases, as they correspond to the expansions at the singularity $\lambda$ (or $\mu$ ) with exponent zero, and thus the above equation (21) is valid, except at the points $z=\lambda$ and $z=\mu$. 


\section{\$8. The Hypergeometric Function.}

As is shown in text books on differential equations, e.g. Forsyth, $\S 117$, the hypergeometric equation admits of 24 particular solutions: we have seen that there are six distinct solutions at various points in the $z$-plane, and consequently these 24 solutions may be collected into six groups of 4 , the four solutions in each group being fundamentally the same.

Thus, for example, adopting the usual notation, we have

$$
y_{1}=y_{2}=y_{17}=y_{18}
$$

and these may be transformed into the corresponding members of the other groups.

Now $y_{1} \equiv \mathrm{F}(a, b, c, z)$,

whence

$\frac{1}{y_{1}} \frac{d y_{1}}{d z}=\frac{-a b}{(a+b+1) z-c}-\frac{(a+1)(b+1) z(z-1)}{(a+b+3) z-(c+1)}-\ldots-\frac{(a+n)(b+n) z(z-1)}{-(a+b+2 n+1) z-(c+2)}-\ldots$

also $y_{2} \equiv(1-z)^{c-a-b} \mathrm{~F}(c-a, c-b, c, z)$,

whence

$\frac{1}{y_{2}} \frac{d y_{3}}{d z}=-\frac{c-a-b}{1-z}+\frac{-(c-a)(c-b)}{(2 c-a-b+1) z-c}-\frac{(c-a+1)(c-b+1) z(z-1)}{(2 c-a-b+3) z-(c+1)}$

$$
\ldots-\frac{(c-a+n)(c-b+n) z(z-1)}{(2 c-a-b+2 n+1) z-(c+n)}
$$

dgain, $\quad y_{17} \equiv(1-z)^{-a} \mathrm{~F}\left(a, c-b, c, \frac{z}{z-1}\right)$,

whence

$$
\begin{array}{r}
\frac{1}{y_{17}} \frac{d y_{17}}{d z}=\frac{a}{1-z}-\frac{1}{z-1}\left\{\frac{-a(c-b)}{(a-b+1) z+c}-\frac{(a+1)(c-b+1) z}{(a-b+2) z+(c+1)}-\ldots \ldots\right. \\
\left.\ldots-\frac{(a+n)(c-z+n) z}{(a-b+n+1) z+(c+n)-\ldots}\right\}
\end{array}
$$

similarly from $y_{18} \equiv(1-z)^{-b} \mathrm{~F}\left(b, c-a, c, \frac{z}{z-1}\right)$, we obtain

$$
\begin{array}{r}
\frac{1}{y_{18}} \frac{d y_{18}}{d z}=\frac{b}{1-z}-\frac{1}{z-1}\left\{\frac{-b(c-a)}{(b-a+1) z+c-\frac{(b+1)(c-a+1) z}{(b-a+2) z+(c+1)}-\ldots}\right. \\
\left.\ldots-\frac{(b+n)(c-a+n) z}{(b-a+n+1) z+(c+n)-\ldots}\right\} \ldots
\end{array}
$$


Now these four solutions $y_{1}, y_{2}, y_{15}, y_{18}$ are identical, and consequently the four continued fractions (23)-(26) derived from them by the same process in all cases are identical.

The hypergeometric series is, however, only convergent in the region $|z|<1$, so that at the point $z=1$ and outside the unit circle enclosing the origin the continued fraction relations given above do not hold. This limitation also applies in the cases of the relations between continued fractions derived from the hypergeometric equation in a later section of this paper.

\section{\$9. Other relations deduced from hypergeometric functions.}

In addition to the identities between hypergeometric functions utilised in the preceding section, several important relations are known connecting different hypergeometric series, which would be expected to give rise to relations involving continued fractions. Thus from an equation given by Gauss, viz.

$$
(1+z)^{2 a} \mathrm{~F}(2 a, 2 a+1-c, c, z)=\mathrm{F}\left(a, a+\frac{1}{2}, c, \frac{4 z}{(1+z)^{2}}\right),
$$

is obtained the relation

$$
\begin{aligned}
& \frac{a}{2(1-z)}-\frac{(1+z)}{4(1-z)}\left\{\frac{2 a(2 a+1-c)}{(4 a+2-c) z-c}-\frac{(2 a+1)(2 a+2-c) z(z-1)}{(4 a+4-c) z-(c+1)}-\ldots\right. \\
& \left.\ldots-\frac{(2 a+n)(2 a+n-c) z(z-1)}{(4 a+2 n+2-c) z-(c+n)}-\ldots\right\} \\
& =\frac{-a\left(a+\frac{1}{2}\right)}{\left(2 a+\frac{3}{2}\right) 4 z-c(1+z)^{2}}+\frac{(a+1)\left(a+\frac{3}{2}\right) 4 z(1-z)^{2}}{\left(2 a+\frac{7}{2}\right) 4 z-(c+1)(1+z)^{2}}+\ldots \\
& (a+n)\left(a+\frac{2 n+1}{2}\right) 4 z(1-z)^{2} \\
& \ldots+\overline{\left(2 a+2 n+\frac{3}{2}\right) 4 z-(c+n)(1+z)^{2}}
\end{aligned}
$$

Another equation given by Gauss, viz.

$$
(1+z)^{2} \mathrm{~F}\left(a, a+\frac{1}{2}-b, b+\frac{1}{2}, z^{2}\right)=\mathrm{F}\left(a, b, 2 b, \frac{4 z}{(1+z)^{2}}\right),
$$

gives rise to the following continued fraction relation 


\section{6}

$$
\begin{aligned}
& \frac{a}{2(1-z)}+\frac{z(1+z)}{2(1-z)}\left\{\frac{-a\left(a-b-\frac{1}{2}\right)}{\left(2 a-b+\frac{3}{2}\right) z^{2}-\left(b+\frac{1}{2}\right)}-\frac{(a+1)\left(a-b+\frac{3}{2}\right) z^{2}\left(z^{2}-1\right)}{\left(2 a-b+\frac{7}{2}\right) z^{2}-\left(b+\frac{3}{2}\right)}\right. \\
& \left.\frac{(a+n)\left(a-b+\frac{2 n+1}{2}\right) z^{2}\left(z^{2}-1\right)}{\left(2 a-b+2 n+\frac{3}{2}\right) z^{2}-\left(b+\frac{2 n+1}{2}\right)}-\ldots\right) \\
& =\frac{-a b}{(a+b+1) 4 z-2 b(1+z)^{2}}-\frac{(a+1)(b+1) 4 z(1-z)^{2}}{(a+b+3) 4 z-(2 b+1)(1+z)^{2}} \\
& \ldots-\frac{(a+n)(b+n) 4 z(1-z)^{2}}{(a+b+2 n+1) 4 z-(2 b+n)(1+z)^{2}}-\ldots
\end{aligned}
$$

Another relation between two continued fractions arises out of an equation given by Kummer, viz.

$$
\mathrm{F}\left(a, b, a+b+\frac{1}{2}, \sin ^{2} \theta\right)=\mathrm{F}\left(2 a, 2 b, a+b+\frac{1}{2}, \sin ^{2} \frac{\theta}{2}\right)
$$

from which we derive the following:-

$$
\begin{aligned}
& \frac{2 a b}{-(2 a+2 b+1) \cos \theta}+\frac{(2 a+1)(2 b+1) \sin ^{2} \theta}{-(2 a+2 b+3) \cos \theta}+\ldots+\frac{(2 a+n)(2 b+n) \sin ^{2} \theta}{-(2 a+2 b+2 n+1) \cos \theta}+ \\
& =\cos \theta\left\{\frac{a b}{\frac{1}{2}-(a+b+1) \cos ^{2} \theta}+\stackrel{(a+1)(b+1) \sin ^{2} \theta \cos ^{2} \theta}{-3}-(a+b+3) \cos ^{2} \theta+\ldots+\frac{(a+n)(b+n) \sin ^{2} \theta \cos ^{2} \theta}{\frac{2 n+1}{2}-(a+b+2 n+1) \cos ^{2} \theta+\ldots}\right\}
\end{aligned}
$$

or if $\cos \theta=z$.

$$
=z\left\{\frac{a b}{\frac{1}{2}-(a+b+1) z^{2}}+\frac{(a+1)(b+1) z^{2}\left(1-z^{2}\right)}{\frac{3}{2}-(a+b+3) z^{2}}+\ldots+\frac{(a+n)(b+n) z^{2}\left(1-z^{2}\right)}{\frac{3 n+1}{2}-(a+b+2 n+1) z^{2}}+\ldots\right\}
$$

when $|z|<1$.

Kummer also gives the following relation

$$
\mathrm{F}\left(\frac{a}{2}, \frac{a+1}{6}, \frac{2 a+2}{3}, \sin ^{2} 2 \theta\right)=\cos ^{-2 a} \theta \mathrm{F}\left(\frac{a}{2}, \frac{a+1}{6}, \frac{2 a+2}{3},-\frac{4 \sin ^{2} \theta}{\cos ^{4} \theta}\right) .
$$


If $y=\mathrm{F}\left(\frac{a}{2}, \frac{a+1}{6}, \frac{2 a+2}{3}, \sin ^{2} 2 \theta\right)$, the continued fraction corresponding to it is

$$
\begin{gathered}
\frac{1}{y} \frac{d y}{d\left(\sin ^{2} 2 \theta\right)}=\frac{\frac{-a(a+1)}{12}}{\left(\frac{2 a}{3}+\frac{7}{6}\right) \sin ^{2} 2 \theta-\frac{2 a+2}{3}+\frac{(a+2)(a+7)}{12} \sin ^{2} 2 \theta \cos ^{2} 2 \theta} \frac{\left.\frac{2 a}{3}+\frac{19}{6}\right) \sin ^{2} 2 \theta-\frac{2 a+5}{3}+\ldots}{\frac{(a+2 n)(a+6 n+1)}{12} \sin ^{2} 2 \theta \cos ^{2} 2 \theta} \\
\ldots+\frac{\left.\frac{12 a}{3}+\frac{12 n+7}{6}\right) \sin ^{2} 2 \theta-\frac{2 a+3 n+2}{3}}{\left(\frac{2 a}{3}+\ldots .\right.} \\
=-\frac{1}{y} \frac{d y}{d\left(\cos ^{2} \theta\right)} \frac{1}{4\left(\cos ^{2} \theta-\sin ^{2} \theta\right)}
\end{gathered}
$$

Also, if $v=\mathrm{F}\left(\frac{a}{2}, \frac{a+1}{6}, \frac{2 a+2}{3},-\frac{4 \sin ^{2} \theta}{\cos ^{4} \theta}\right)$ then the corresponding continued fraction is

$$
\begin{aligned}
& \frac{1}{v} \frac{d v}{d\left(\frac{-4 \sin ^{3} \theta}{\cos ^{4} \theta}\right)}=\frac{\frac{-a(a+1)}{12}}{-\left(\frac{2 a}{3}+\frac{7}{6}\right) \frac{4 \sin ^{2} \theta}{\cos ^{4} \theta}-\frac{2 a+2}{3}}-\frac{\frac{(a+2)(a+7)}{12} \frac{4 \sin ^{2} \theta\left(\cos ^{2} \theta-2\right)^{2}}{\cos ^{8} \theta}}{-\left(\frac{2 a}{3}+\frac{19}{6}\right) \frac{4 \sin ^{2} \theta}{\cos ^{4} \theta}-\frac{2 a+5}{3}}-\ldots \ldots \ldots \\
& \ldots-\frac{\frac{(a+n)(a+6 n+1)}{12} \cdot \frac{4 \sin ^{2} \theta\left(\cos ^{2} \theta-2\right)^{2}}{\cos ^{8} \theta}}{-\left(\frac{2 a}{3}+\frac{12 n+7}{6}\right) \frac{4 \sin ^{2} \theta}{\cos ^{4} \theta}-\frac{2 a+3 n+2}{3}}-\ldots \\
& =-\frac{1}{4 v} \frac{d v}{d\left(\cos ^{2} \theta\right)} \frac{\cos ^{6} \theta}{\cos ^{2} \theta-2} .
\end{aligned}
$$$$
\text { Now } \frac{1}{y} \frac{d y}{d\left(\cos ^{3} \theta\right)}=\frac{-a}{\cos ^{2} \theta}+\frac{1}{v} \frac{d v}{d\left(\cos ^{2} \theta\right)}
$$

from which we may deduce another continued fraction relation, viz. 


$$
\begin{aligned}
& \begin{aligned}
-4 \cos 2 \theta\left\{\frac{\frac{a(a+1)}{12}}{\left(\frac{2 a}{3}+\frac{7}{6}\right) \sin ^{2} 2 \theta-\frac{2 a+2}{3}}+\right. & \frac{\frac{(a+2)(a+7)}{12} \sin ^{2} 2 \theta \cos ^{2} 2 \theta}{\left(\frac{2 a}{3}+\frac{19}{6}\right) \sin ^{2} 2 \theta-\frac{2 a+5}{3}+\ldots \ldots \ldots \ldots \ldots \ldots .} \\
& \left.+\frac{(a+2 n)(a+6 n+1) \sin ^{2} 2 \theta \cos ^{2} 2 \theta}{\left(\frac{2 a}{3}+\frac{12 n+7}{6}\right) \sin ^{2} 2 \theta-\frac{2 a+3 n+2}{3}}+\ldots \ldots \ldots \ldots\right\}
\end{aligned} \\
& =\frac{a}{\cos ^{2} \theta}+\frac{\cos ^{2} \theta-2}{\cos ^{2} \theta}\left\{\frac{\frac{-a(a+1)}{3}}{\left(\frac{2 a}{3}+\frac{7}{6}\right) 4 \sin ^{2} \theta-\frac{2 a+2}{3} \cos ^{4} \theta-\frac{\frac{(a+2)(a+7)}{3}}{-\left(\frac{2 a}{3}+\frac{19}{6}\right) 4 \sin ^{2} \theta-\left(\frac{2 a+3}{3}\right) \cos ^{4} \theta}}\right. \\
& \left.-\ldots-\frac{\frac{(a+n)(a+6 n+1)}{3}}{-\left(\frac{2 a}{3}+\frac{12 n+7}{6}\right) 4 \sin ^{2} \theta-\left(\frac{2 a+3 n+2}{3}\right) \cos ^{4} \theta-\ldots}\right\} \text {. }
\end{aligned}
$$

All the relations between continued fractions which are derived from equations between hypergeometric functions must be identically true, since an equation between the hypergeometric functions implies that they are the same solution of the same differential equation.

In conclusion, I must thank Professor Whittaker, at whose instigation this present research was begun, for encouragement and advice given to me during its progress. 